\title{
Litiasis urinaria en el niño
}

\author{
DRES. F. PUGa *, S. PAREDES *, H. MAGLIIRE*, A. MOREY * C. IzZO*, SRA. H. KUMSACK *.
}

INTroducción. La Litiasis Urinaria infantil es de escasa frecuencia, en relación con otras enfermedades nefrourológicas, como lo demuestran las pocas publicaciones nacionales y extranjeras. $(1,2,3,4)$.

Sin embargo, su diagnóstico es indispensable para realizar una terapia racional que estará supeditada a la ubicación y tamaño de los cálculos. (5).

En el niño la mayoría de las litiasis se presentan ya sea con dolores, a veces con caracteres de cólicos renales, o bien aparecen en aquellos pacientes con infecciones urinarias recidivantes o hematurias recidivantes o hematurias transitorias (6).

La presente publicación se refiere a 43 niños estudiados en el Hospital Luis Calvo Mackenna y en los cuales se encontró litiasis de la vía urinaria.

Material y MÉtodó. Se analizan 43 casos de enfermos con Litiasis de la vía urinaria atendidos en el Hospital L. Calvo Mackenna entre los años 1955 y 1971. De ellos 8 son del sexo femenino $(18,6 \%)$ y 35 del sexo masculino $(81,4 \%)$ y sus edades se distribuyen de acuerdo con la figurn Nọ 1 , siendo el de menor edad de ellos, de dos meses.

En todos los pacientes se confirmó la sospecha clínica de litiasis urinaria mediante una piclografía de climinación.

En 23 pacientes se determinó la composición química del cálculo obtenido, y en 18 se realizó un análisis bioquímico que se detalla a continuación: Cromatografía bidimensional en papel de la orina. calcemia, fosfemia, excreción de calcio y fósforo en 24 horas por la orina; clearence de creatinina endógena; prueba de concenttración con determi-

\footnotetext{
* Depto. Nefrourología, Hospital L. Cal*o Mackenna.

Laboratorio Central, Hospital L. Calvo Mackenna.
}

nación de osmolaridad máxima; excreción de hidrogeniones después de una sobrecarga de amonio; acidez titulable y ph en la orina mediante papel tornasol, repetido varias veces al día.

En 23 pacientes $(53,4 \%)$ se encontró malformación urológica.

Una vez hecho el diagnóstico, en todos los pacientes fue necesaria la intervención quirúrgica, excepto en $6(13,9 \%)$ que eliminaban espontáneamente el cálculo.

A 12 de los niños intervenidos se les practica una Biopsia Renal durante el acto operatorio. En 6 pacientes fue necesario una reintervención por la repetición de la Litiasis.

ResUctados a) anamnesis: el motivo por el cual estos pacientes acuden a consultar es habitualmente en referencia a alguna manifestación de la vía urinaria.

Las causas de consulta pueden verse en la tabla No 1. En ellos podemos apreciar que alrede-

TA B L A No 1

LITIASIS URINARIA, SINTOMAS, SIGNOS Y HECHOS MAS NOTORIOS

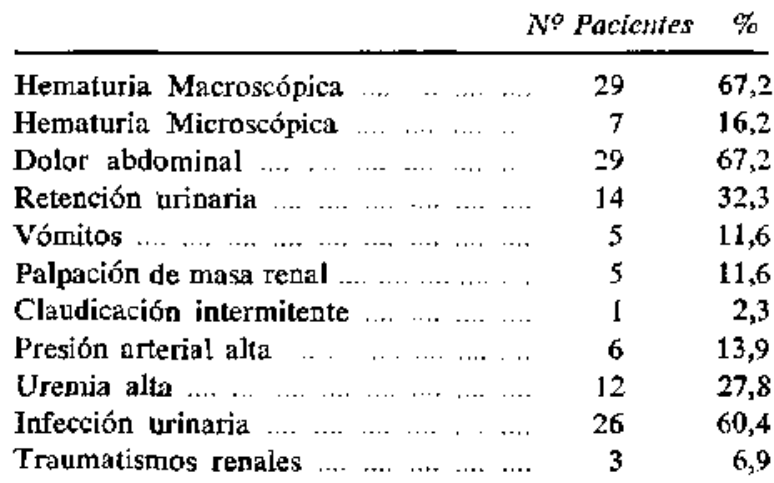


dor de las $2 / 3$ partes consultan por dolores abdominales, cólicos habitualmente no característicos, aunque en ciertos casos presentan las manifestaciones típicas de dolor lumbar irradiadas a la zona genital o muslo. No es raro que el motivo de consulta sea por dolor testicular. Con frecuencia estos cuadros cólicos se presentan acompañados de hematuria macroscópica transitoria de sangre roja.

La segunda causa de consulta por la cual se pesquisa la Litiasis es la infección urinaria recidivante lo que hace practicar un estudio urológico completo.

Por otra parte, no es raro que el diagnóstico de Litiasis se haga porque el niño traiga el cálculo, eliminado espontáneamente.

Como causa menos frecuente de diagnóstico de litiasis está la aparición de una masa renal palpable dolorosa que corresponde a una hidronefrosis aguda por obstrucción; hematurias que corresponden a traumatismos abdominales mínimos $\mathrm{y}$, por último es jmportante destacar que en dos de muestros pacientes el hallazgo de Litiasis Renal fue absolutamente casual por un estudio que nada tenía que ver con la vía urinaria.
La litiasis vesical (Figura No 2) que observamos en 14 de nuestros pacientes (34\%) se presenta en forma bastante característica: se inicia la micción con un chorro normal el que bruscamente se interrumpe y a pesar de los esfuerzos del niño no puede continuar, hasta después de un período de reposo, especialmente en decúbito. Casi siempre se acompaña de dolor hipogástrico, hematuria macroscópica y de infección urinaria.

b) antecedentes personales y familiares: en cuanto a los antecedentes encontrados en estos pacientes (Tabla No 2) hay que destacar la excesiva ingesta de Vitamina $D$ ( 5 pacientes) que fue confirmado mediante la determinación de calciuria en 24 horas cuyos valores estaban elevados en todos estos pacientes.

Las deshidrataciones que son frecuentes en nuestro medio también serían un agente coadyudante en la etiología de la Litiasis urinaria. (8 pacientes).

También son importantes los antecedentes de fracturas, inmovilizaciones prolongadas o Poliomielitis invalidante.

En sólo 3 pacientes se encontró antecedentes familiares de Litiasis.

J०Paclentes

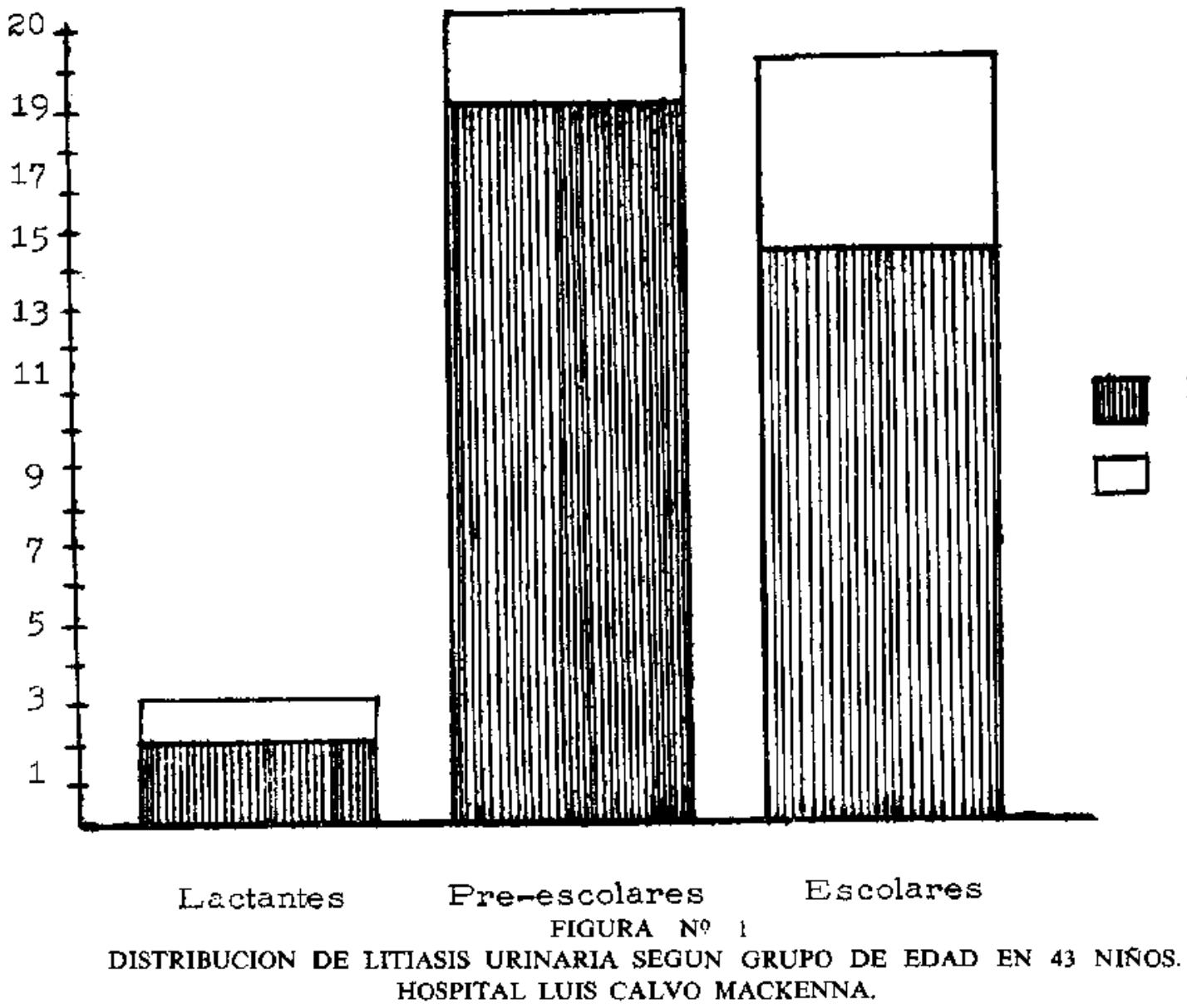




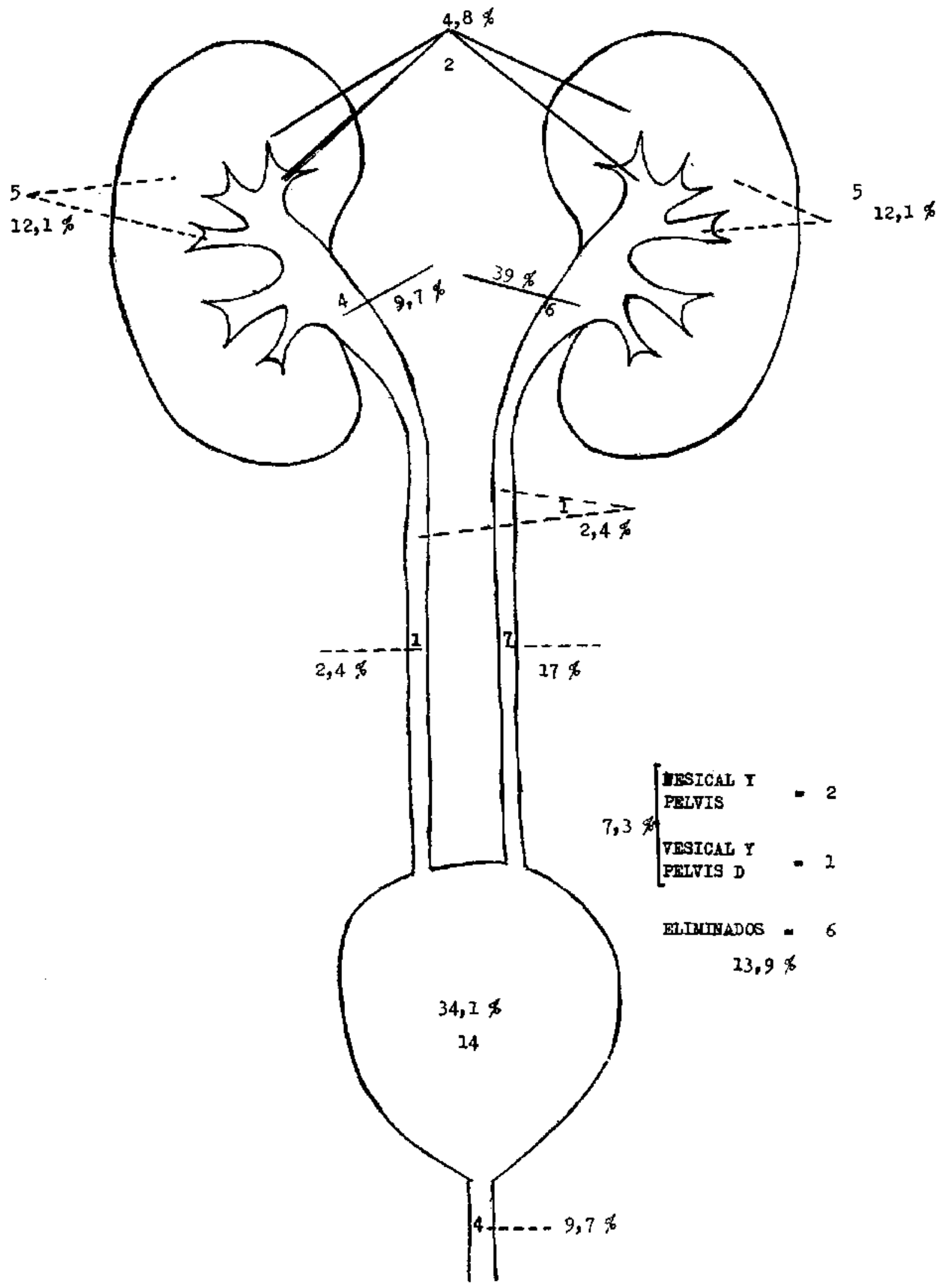

FIGURA No 2

LITIASIS URINARIA. SITIO Y NUMERO DE CALCULOS. 
TABLA No 2

LITIASIS URINARIA. HALLAZGOS EN ANAMNESIS $Y$ EXAMEN FISICO

No pacientes

Excesiva ingesta. Vit. D2

5

Fracturas e inmovilización

Deshidrataciones previas

8

Parasitosis intestinal masiva

1

Fimosis

4

Sindrome pluricarencial

1

Poliomelitis previa

1

Ingesta excesiva de sulfas (Sulfatiazol)

Antecedentes familiares de litiasis renal ...

Espina Bifida

c) ubicación: la ubicación de los cálculos encontrados en los 43 pacientes de acuerdo a la pielografía de eliminación y a los hallazgos quirúrgicos pueden observarse en la figura $N^{\circ} 2$.

Se encontraron 4 Litiasis uretrales $(9,7 \%)$, 14 Vesicales $(34,1 \%), 8$ uretrales $(19,4 \%), 10$ calicilares $(28,7 \%)$, y 10 piélicas $(28,7 \%)$.

En sólo 3 casos los cálculos fueron bilaterales ( 2 renales y 1 ureteral).

d) Iropatia obstructiva: a 29 pacientes se les practicó un estudio urológico completo, encontrándose uropatía obstructiva asociada en 23 de ellos (79\%) (Tabla No 3).

En los 6 pacientes reintervenidos $(13,9 \%)$ por repetición de la Litiasis se obtuvieron los siguientes hallazgos: uropatía obstructiva en 5 de eilos, $y$ nefrocalcinosis en 1 .

\section{TABLA No 3}

MALFORMACIONES ENCONTRADAS EN CUAREN -

TA Y TRES (43) PACIENTES CON LITIASIS URINARIA

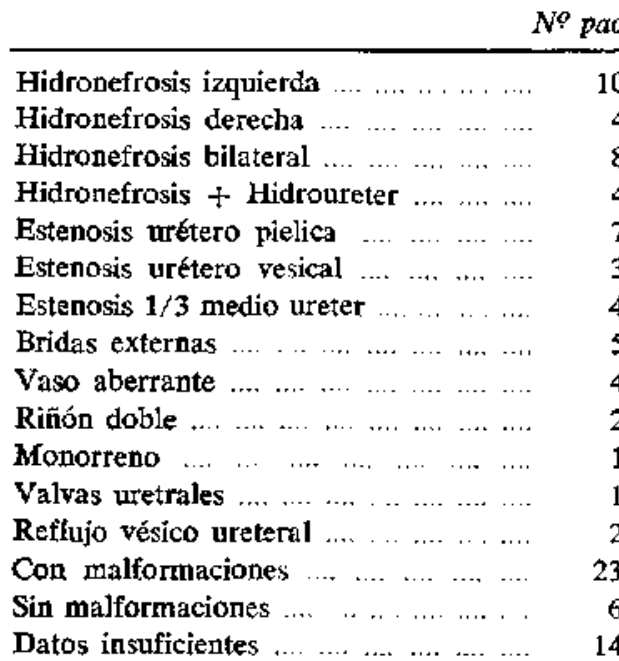

\section{0}

4

8

4

7

3

4

5

4

2

1

1

En esta experiencia, se sometió a intervención quirúrgica a 37 pacientes $(86 \%)$, fue necesario practicar 7 nefrectomías $(18,9 \%)$ por lo avanzado đel daño renal, 14 plastías pieloureterales, fuera de ottas intervenciones que consistieron en la corrección de la obstrucción por vaso aberrante o por adberencias pieloureterales.

e) estudio químico del cálculo: el estadio de los cristales en el sedimento urinario no nos ha servido como elemento que sea congruente con la composición química del cálculo extirpado, lo que obliga al estudio químico de éste, el que se realizó cn 23 pacientes $\left(53,4 \%\right.$ ) (Figura $N^{\circ} 3$ ).

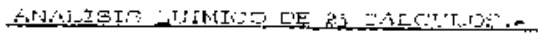

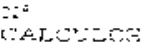

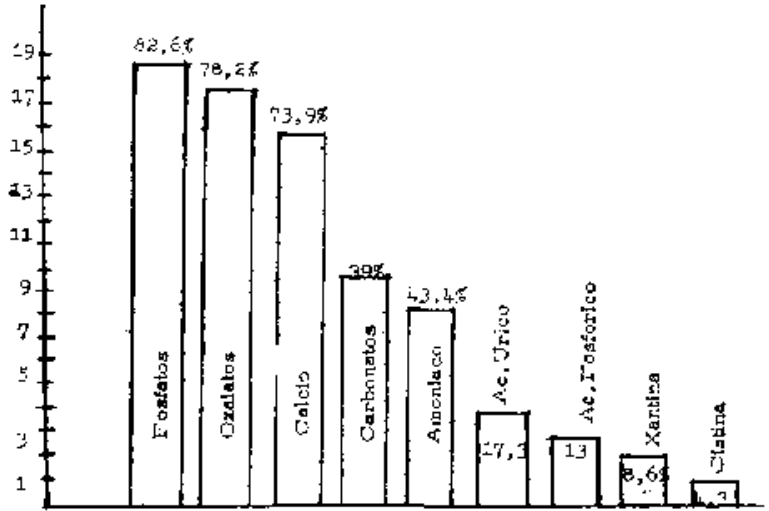

Se confirmó en la mayoría de los casos una naturaleza mixta ( $87 \%$ ) seguido de las combinaciones de amonio, ácido úrico, xantina y cistina.

f) estudios bioquímicos: con el fin de pesquisar una posible patología tubulàr causante de la litiasis se hizo primero cromatografía bidimensional en papel de la orina en 13 niños $(30,2 \%)$ (Tabla Nơ 4), ésta resultó normal en $4(30,8 \%)$.

TALIA NQ 4

\section{CROMATOGRAFIA BIDIMENSIONAL REALIZADA EN TRECE (13) PACIENTES CON LITIASIS URINARIA}

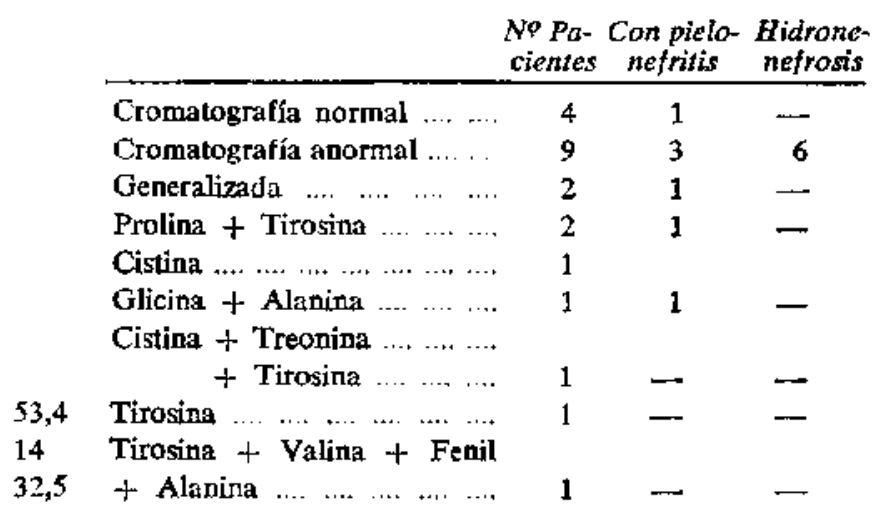


De los 9 pacientes restantes en que cl resultado fue mortal, en 8 se encontró una pielonefritis $(88,9 \%$ ) (en 4 confirmada mediante biopsia renäl), el otro paciente tenía un exceso de cistina en la orina, depósito de cistina en la córnca y un compromiso en la función renal acentuados.

Desgraciadamente este paciente, con el presunto diagnóstico de cistinosis no ha sido posible encontrarlo para proseguir su estudio. El análisis químico del cálculo de ese paciente no reveló existencia de cistina.

En 18 pacientes $(41,8 \%)$ se realizó un estudio de acidificación' de la orina cuyos resultados pueden verse en la tabla Nọ 5 .

TABLA No 5

PROMEDIOS NORMALES ENCONTRADOS EN NINOS CON LITIASIS URINARIA DESPUES DE SOBRECARGA CON $\mathrm{NH}_{4} \mathrm{CL}$

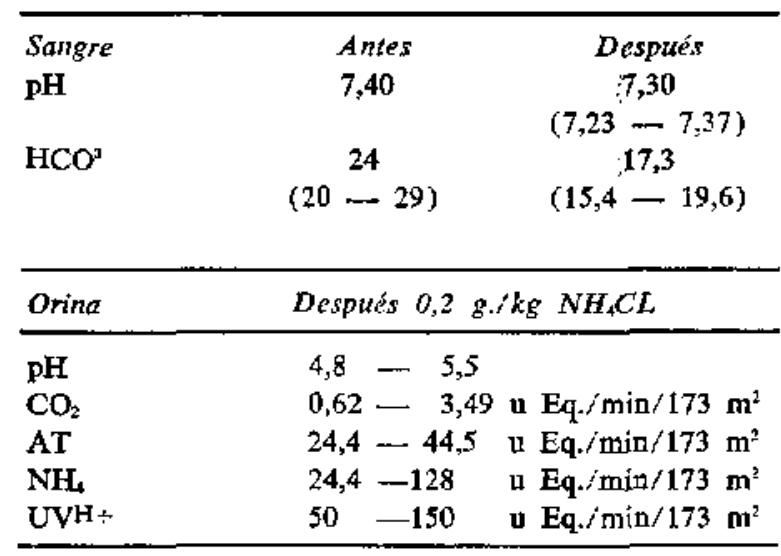

Amonoigénesis y la excreción de hidrogeniones después de una sobrecarga de cloruro de amonio de 0,2 gr. por kilo se observa normal con baja del ph sanguíneo de 7,40 a 7,30 y del $\mathrm{CO}^{2}$ total de 24 a 17 miliequivalentes por litro.

Sólo 4 pacientes, con un clearence de creatinina discretamente bajo, tuvieron una acidez titulable que no sobrepasó los 20 miliequivalentes y una excreción de amonio de 39 miliequivalentes. En todos los casos, la capacidad de concentración urinaria fue insatisfactoria.

En cuanto al manejo del fósforo y considerando sólo 5 pacientes que turvieron hipercalciuria, puede verse que 3 tienen un porcentaje de absorción tubular máximo inferior al normal y que también el fósforo total filtrado está bajo lo normal (Tabla No 5). Y, naturalmente la relación excreción de calcio de cretatinina está francamente aumentada como era de esperar en todos aquellos pacientes con excreción de calcio aumentada en las 24 horas.
Comentario. Con los resultados expuestos, se puede deducir que el interrogatorio acucioso es importante en el estudio de los enfermos de litiasis. Aparte de la búsqueda de antecedentes familiares de litiasis renal debe investigarse la ingestión de Vitamina $D$, habitualmente recetada en forma generosá en los consultorios de niños sanos; antecedentes de fracturas, inmovilizaciones prolongadas, secuelas de poliomielitis invalidante; las deshidrataciones que son frecuentes en nuestro međio también serían un agente coadyudante en la etiología de Litiasis Urinaria. Tanto la excesiva administración de Vitamina $\mathrm{D}$ en el período de lactantes como las inmovilizaciones $y$ secuelas de poliomielitis actuarían a través de un mecanismo de producción de hipercalciuria, la que puede ser transitoria, o bien continuar por varios meses después de suspendida la causa, sobre todo en el caso de la Vitamina D, la que se acumula en el organtismo y no se elimina (7).

Nos parece que estos resultados nos obligan a reactualizar los csquemas establecidos por el Servicio Nacional de Salud para el control de niños sanos en lo que se refiere a la administración de esta vitamina en los primeros años de vida.

En cuanto a la deshidratación y su relación con la aparición de la litiasis, intervendría aumentando la concentración de la orina, y pernitiendo el depósito de cristales en la vía urinaria.

Vimos que entre los motivos de consulta o de pesquisa de litiasis, que muchos de ellos eran por infección urinaria recidivante. Vale la pena destacar que la litiasis es excepcional en las infecciones urinarias tomadas en total $(1,2 \%)$, pero que por el contrario esta infección se presenta en el $82,7 \%$ en los pacientes con litiasis. Su relación de causa-efecto con la litiasis es muy discutida. Se ha planteado la posibilidad de que Jos detritus celulares y bacterias formaran el núcleo originario del cálculo, hipótesis que no ha sido demostrada (5). Otro factor, a través del cual intervendría la infección urinaria, sería a través de las modificaciones del ph urinario, to que favorecería la precipitación de sales. A 19 pacientes se les analizó el ph de la orina en forma repetida con papel tornasol. En 13 de ellos el ph fue inferior a 6 y en 6 solamente superior a éste. Sin embargo, 12 de estos pacientes tenían una infección urinaria siendo la distribución de cllas muy similar en ambos grupos. Vale la pena destacar que las orinas ácidas favorecerían la formación de cálculos úricos y cistínicos y las alcalinas en su mayoría corresponderíana a cálculos de fosfatos de calcio, fosfato de amonio magnesiano $y$ en cualquier ph se formarían los cálculos de oxalato de calcio. (8).

El alto porcentaje de uropatía obstructiva en un $80 \%$ en los niñes a los que se les practicó un estudio urológico completo, hace concluir que la ectasia es indudablemente un factor favorecedor 
del depósito de cristales en pacientes que también tienen una infección urinaria. $(5,6)$.

Está de más insistir que en el acto operatorio no debe solamente hacerse la litotomía, sino que es indispensable reparar la obstrucción, que habitualmente se encuentra en la vía urinaria.

El diagnóstico y el tratamiento debe hacerse precozmente a fin de evitar las graves consecuencias que acarrea esta patología, ya que seguramente la lesión pielonefrítica se instala rápidamente en el riñón afectado. De allí la necesidad de contar con un servicio especializado que pesquise y controle a este grupo de niños.

\section{Resumen}

Se analizan 43 pacientes portadores de Litiasis Urinaria. En 41 se hizo extracción quirúrgica y en dos éstos fueron eliminados espontáneamente.

Las edades fluctuaron entre dos meses quince dias y quince años distribuyéndose en iguales proporciones en Pre-escolares y escolares, con franco predominio en el sexo masculino.

En el 53,4\% se encontró una malformación urológica obstructiva $y$ en el $82,7 \%$ asociado a infección urinaria.

La Litiasis fue recidivante en el $14 \%$ en pacientes en que no fue solucionado el problema obstructivo.

$E_{n} 23$ enfermos se hizo estudio químico del cálculo y en 18 estudio metabólico del paciente.

Se comenta el abuso del "Golpe Vitamínico" asociado o no a ingestión excesiva de Calcio.

En doce enfermos se hizo biopsia renal quirúrgica durante el acto operatorio, encontrándose en el $82,3 \%$ lesiones compatibles de Pielonefritis.

Se discuten las probabilidades etiológicas de litiasis urinaria.

\section{SUMMARY}

The records of 43 children suffering Calculous disease of the Urinary tract are analized.

41 underment surgery and 2 were eliminated spontaneously.

Ages variated from 2 months to 15 years with equal number of pre school-school ( 6 years) attending children ( -6 years) Males predominated. $53,4 \%$ had an obs- tructive congenital malformation of the urinary tract and $82,7 \%$ had urinary infection.

Calculosis recurred in $14 \%$ the patients, thease had untreated obstructive condition.

Chemical analisis of calculi was done in 23 cases and 18 had metabolic study.

Abuse of Vit. D. administration with or without Calcium is commented as etiologie factor of some calculosis.

12 renal biopsias showed $82,3 \%$ of pielonephritis. Various causes of urinary Lithiasis are discused.

\section{Bibliografía}

1.-James E. Went MD; Edmund C. Burke MD; Gunhar B. Silkter MD; and Davis C, Utz MD. Nephrolitiasis and Nephrocalcinosis in children. Pediatrics vol. 41: $57,1968$.

2.-C. W. Daeschner, MD.; E. D. Singleton, MD; and C. Curtis MD. Urinary tract calculi and Nephrocalcinosis in infants and children. The Journal of Pediatrics vol. $57: 751,1960$.

3.- Jorge Litvak L., Eduardo Talloman V. Litiasis Renal. Rev. Med. Chile vol. 290: 1960.

4.-R. Gantes y A. Guzmán. Ĺtiasis Urinaria "Casos Clínicos" Arch. Hosp. Niños Roberto del Río. vol. 4-36-38-70-73, 1933.

5.-D. Innes Wilhams and H. B. Eckskeiw. Urinary Lithirsis.

6.- N. A. Myers. Urolithiasis in childhood. Arch, Diseases in Childhood. vol. 32-47, 1957.

7.-Henneman P. H. y Baker W. H. The Mechanism of sustained hypercalcemia, Following hipervitaminosis $\mathbf{D}$, and the Milkalkali Syndrome. J. Clinic Inv. vol. $36-899,1957$.

8.-Edwin L. Prsent. MD. and Edwin L. Prien Sr. MD. Composition and Structure of Urinary Stong American. American Journal of medicine vol. 45-664, 1968.

9.-M. R. Wills. The Urinary Calcium Creatinine Ratio As. A. Measure of Urinary Calcium Excretion. J. Clinic Path. vol. 22-287, 1969. 